\title{
Beams in Magnetised Pair Plasmas
}

\author{
E. T. Rowe \\ Special Research Centre for Theoretical Astrophysics, School of Physics, \\ Sydney University, NSW 2006, Australia
}

\begin{abstract}
The radiative instability was considered in the context of pulsar radio emission by Goldreich \& Keeley (1971) and rederived and extended by Asséo, Pellat, \& Sol (1983) and by Asséo (1995). Their results can be generalised and reproduced by replacing the thin cylindrical ring of charged particles by an infinitesimally thin current carrying sheet in either planar or cylindrical geometry, suggesting that the local ring geometry may not be essential for the instability. It may be useful to further investigate the generalised dispersion equation given below.
\end{abstract}

\section{Introduction}

In the Goldreich and Keeley model, instability occurs for charges moving around a thin cylindrical ring bounded by vacuum. Buschauer \& Benford (1978) suggested that this is due to the acceleration of particles around the ring. Asséo et al. and Asséo model the ring as a strongly magnetised pair plasma annulus, and obtain a dispersion equation by enforcing boundary conditions on the fields at the edge of the annulus. Asséo et al. argue that sharp boundaries are essential for instability, particle motion along curved magnetic field lines alone being insufficient. Rowe \& Rowe (1999a) considered wave modes for a thin pair plasma slab in planar geometry and this work implied a connection between the planar and cylindrical cases. We consider an infinitesimally thin current carrying interface (beam) between bounding plasmas and obtain an analytic dispersion relation for wave perturbations which is independent of the geometry. This result contains and generalises those of both the planar geometry and cylindrical geometry cases, and is obtained in a straightforward manner.

\section{General Thin Beam Dispersion Relation}

The general dispersion equation is derived in a recently submitted article (Rowe $\&$ Rowe 1999b). We consider a beam of electrons and positrons flowing with speed $U$ along a strong magnetic field relative to bounding electron-positron plasmas with different plasma frequencies. We use a coordinate system, not necessarily cartesian, with basis vectors $(\hat{\boldsymbol{x}}, \hat{\boldsymbol{y}}, \hat{\boldsymbol{z}})$, where $\hat{\boldsymbol{x}}$ is normal to the beam interface and $\hat{\boldsymbol{y}}$ is parallel to the magnetic field. Dielectric tensors in each medium 
have the form $\boldsymbol{K}(\omega, \boldsymbol{k})=(\boldsymbol{W}-1) \hat{\boldsymbol{y}} \hat{\boldsymbol{y}}+\delta$, with $\delta$ the unit tensor and

$$
W=1-\frac{\omega_{p}^{2}}{\gamma^{3}\left(\omega-k_{y} U\right)^{2}},
$$

where $\gamma$ is the Lorentz factor of the flow and $\omega_{p}$ the plasma frequency. For the beam $\gamma=\gamma_{p}$ and $\omega_{p}=\omega_{b}$, say, while for the bounding plasmas, $U=0, \gamma=1$ and $\omega_{p}=\omega_{l}$ and $\omega_{r}$.

The induced beam current is

$$
J_{s}(\omega)=i \varepsilon_{0} \omega a\left(1-W_{b}\right) E_{s y}(\omega) \hat{\boldsymbol{y}}
$$

with $E_{s y}(\omega)$ the average of the $y$ component of the electric field across the beam (of width $a$ ) and $W_{b}$ the dielectric constant in the beam. This current leads to a discontinuity in the magnetic fields across the beam but the surface components of the electric field are continuous. The dispersion equation obtained from these boundary conditions has approximate analytical solutions if $\delta=a / x_{0} \ll 1$ (where $x_{0}$ is a suitable scale length), of the form $\omega \sim \omega_{R}+\omega_{\frac{1}{2}} \delta^{\frac{1}{2}}$ where $\omega_{R}=k_{y} U$ and

$$
\omega_{\frac{1}{2}}^{2}=\frac{\omega_{b}^{2} B^{r(0,1)}\left(\omega_{R}, \rho\right) B^{l(0,1)}\left(\omega_{R}, \rho\right)}{\gamma_{p}^{3}\left[W_{l} B^{l}\left(\omega_{R}, \rho\right) B^{r(0,1)}\left(\omega_{R}, \rho\right)-W_{r} B^{r}\left(\omega_{R}, \rho\right) B^{l(0,1)}\left(\omega_{R}, \rho\right)\right]},
$$

with dielectric constants $W_{l}$ and $W_{r}$ and magnetic fields $B^{r}$ and $B^{l}$ in the bounding plasmas evaluated at $\omega=\omega_{R}, \rho=x / x_{0}$ the dimensionless position of the sheet surface and parentheses notation used to denote partial derivatives.

\section{Discussion}

This general result depends only on the values and derivatives of the magnetic fields and dielectric constants in the bounding plasmas, at the interface, and has been shown (Rowe \& Rowe 1999b) to reproduce previous results of planar and cylindrical geometry with the appropriate choice of the magnetic field values. In particular, we can rederive the radiative instability in a local planar geometry, without particle motion along curved trajectories. The instability is of reactive type and can be attributed to phase bunching of particles in the wave. The result suggests that only the magnetic field values and derivatives at the interface determine (in)stability and as these values are unknown for the pulsar application, it may be of interest to investigate it further for various choices.

\section{References}

Asséo, E. 1995, MNRAS, 276, 74

Asséo, E., Pellat, R., \& Sol, H. 1983, ApJ, 266, 201

Buschauer, R., \& Benford, G. 1978, MNRAS, 185, 493

Goldreich, P., \& Keeley, D. A. 1971, ApJ, 170, 463

Rowe, G. W., \& Rowe, E. T. 1999a, J. Plas. Phys. (in press)

Rowe, G. W., \& Rowe, E. T. 1999b, Aust. J. Phys. (submitted) 